\title{
Transient Response of Vertical-Cavity Surface-Emitting Lasers of Different Active-Region Diameters
}

\author{
O. Buccafusca, Member, IEEE, J. L. A. Chilla, J. J. Rocca, P. Brusenbach, and J. Martín-Regalado
}

\begin{abstract}
The multimode dynamics of vertical-cavity surfaceemitting lasers with different active-region diameters was measured under subnanosecond electrical excitation (800-ps pulse duration, 100-ps risetime). The dynamics is characterized by the delayed onset of higher order modes which have a turn-on delay that is dependent on the active-region diameter and the excitation parameters. A simple model that can be used to estimate this turn-on delay for large-area devices is presented. Polarizationresolved measurements show that, under this fast excitation condition, both orthogonal polarization states are isomorphic. The influence of the observed dynamics on the relative intensity noise of these devices is also discussed.
\end{abstract}

Index Terms-Laser modes, multimode dynamics, semiconductor lasers, surface-emitting lasers.

\section{INTRODUCTION}

$\mathbf{T}$ HE RAPID progress achieved in improving the performance of vertical-cavity surface-emitting lasers (VCSEL's) now makes possible their use in applications such as optical data communications [1], [2]. Transient effects associated with high-speed data transfer can produce fast changes on the spatial distribution of the output power of semiconductor lasers. Therefore, the understanding of these effects is essential in applications that include high-speed current modulation. Previous reports on the fast response of VCSEL's have shown that the carrier-photon dynamics of these devices are essentially different from that of edge emitters [3]-[11]. These studies concentrated on the multitransverse mode dynamics of devices with small activeregion diameters. However, larger VCSEL's, with activeregion diameters of the order of $25 \mu \mathrm{m}$, are favored for short-distance optical links [12], [13]. These wide-area devices present more complex multimode dynamics, especially during fast modulation.

Manuscript received June 22, 1998; revised December 15, 1998. This work was supported by the Center for Optoelectronics Computer Systems through the National Science Foundation under Grant EEC-9015128 and by the Colorado Advanced Technology Institute under Grant GEA 95-0002. The work of J. Martín-Regalado was supported by CICYT (Spain) under Project TIC95/0563.

O. Buccafusca, J. L. A. Chilla, and J. J. Rocca are with the Department of Electrical Engineering, Colorado State University, Fort Collins, CO 80523 USA.

P. Brusenbach is with the Vixel Corporation, Broomfield, CO 80021 USA

J. Martín-Regalado is with the Department de Física, Universitat de les Illes Balears and Instituto Mediterráneo de Estudios Avanzados, IMEDEA (CSICUIB), E-07071 Palma de Mallorca, Spain.

Publisher Item Identifier S 0018-9197(99)02557-9.
In this paper, we investigate the transient multimode dynamics of VCSEL's as a function of active-region diameter (up to $24 \mu \mathrm{m}$ ). The study includes temporally and spectrally resolved measurements of the VCSEL output for the two dominant polarization states as well as the determination of the relative intensity noise (RIN) levels. Our results show that higher order modes oscillating within the cavity appear with a time delay that can be estimated utilizing a simple model. A correlation of the observed dynamics with low-frequency RIN measurements showed that larger devices present lower noise levels, especially when operated at high pumping levels. In addition, we studied the polarization properties of the transient response. It had been shown that these devices usually present a dominant polarization state during $\mathrm{dc}$ operation [14], [15] and that the characteristic time for achieving this state is of the order of a few nanoseconds [16], [17]. Our measurements complement this knowledge, showing that the two polarization states of all the transverse modes are present during subnanosecond excitation and that they evolve with the same dynamics.

The various experimental setups and procedures utilized to conduct the measurements are described in the next section. The results are then presented in Section III, which also includes a discussion of the physical processes behind the observed dynamics. Section IV discusses a simple model to estimate the turn-on delay of the higher order transverse modes.

\section{EXPERIMENTAL SETUP}

The lasers used in this study were gain-guided AlGaAs-GaAs multiple-quantum-well (MQW) VCSEL's with cavity mirrors consisting of AlAs-AlGaAs distributed Bragg reflectors (DBR's), which had 19 periods for the p-doped top mirror and 29 periods for the $n$-doped bottom mirror. Measurements were performed in cylindrically symmetric devices with four different active-region diameters: 15, 18, 22 , and $24 \mu \mathrm{m}$ and a top contact window diameter of 12,15 , 18 , and $20 \mu \mathrm{m}$, respectively. In this paper, these devices are identified by their active region size.

To measure the multimode dynamics, the output of the VCSEL was mixed in a lithium iodate crystal with a reference train of 100 -fs pulses from a Ti:sapphire laser, and the resulting sum-frequency signal was detected using a photomultiplier and standard lock-in techniques. The setup was similar 
to the one presented in [8]. The VCSEL's were prebiased through a bias tee and then electrically pumped using a fast pulse generator capable of providing square pulses of 800 ps in duration with 100-ps risetime. Prebias currents were varied from $40 \%$ to $85 \%$ of the dc threshold current $\left(I_{\mathrm{th}}\right)$, while current pulse amplitudes $\left(I_{p}\right)$ ranging from 0.5 to 10 times $I_{\text {th }}$ were utilized to pump the VCSEL. The pulsed current amplitudes were measured with a fast induction current probe (rise and fall times $<100 \mathrm{ps}$ ) constructed specifically for this type of measurement. The VCSEL was operated at a repetition rate of $80 \mathrm{MHz}$ and the electrical pulses were synchronized with the optical pulses of the Ti: sapphire reference beam. By varying the delay between the electrical pulses and the reference beam, the time evolution of the VCSEL output was obtained. The zero delay between the reference optical pulse and the electrical excitation pulse could only be determined within the risetime of the electrical pump - that is, with a resolution of \pm 100 ps. Therefore, the time delays $\left(T_{d}\right)$ were determined relative to the onset of laser emission arbitrarily chosen as the time origin in Figs. 1-4. The time resolution, limited by the jitter of the pulse generator, the synchronization circuitry, and the VCSEL itself, was measured to be $\leq 1.5$ ps. Spectral resolution was achieved by incorporating a monochromator of half-meter focal length to select the wavelength of the VCSEL output before sumfrequency generation. In this way, the different transverse modes, which have different wavelength emission, can be time-resolved. The time-integrated beam profile corresponding to each transverse mode was recorded with a CCD camera placed at the output of the monochromator.

RIN measurements were performed utilizing a setup similar to the one described in [18]. The VCSEL output was modulated by a low-frequency $(\sim 1 \mathrm{kHz})$ optical chopper and detected using a fast photodetector (100-ps rise- and fall-times). The noise component correlating to the chopper frequency was measured with a spectrum analyzer and a lockin amplifier. The spectrum analyzer was set to the zero-span mode in which it acts as a tunable receiver that demodulates the noise signal at a given frequency. This noise signal is then measured by the lock-in amplifier locked to the chopper frequency. By changing the center frequency of the spectrum analyzer, the noise spectrum can be recorded. The average photocurrent was also detected utilizing a lock-in amplifier (without the spectrum analyzer) with suitable integration constants. The advantage of this technique is to avoid the limitations imposed by the noise of the photodetection system, as only signals correlating to the chopper frequency are detected, while uncorrelated signals (photodetector noise, thermal noise in amplifiers, etc.) are rejected. The VCSEL pumping conditions for the RIN measurements were matched to those of the multimode dynamics to allow comparison of the results. Consequently, tuning the spectrum analyzer to frequencies related to the excitation pulse repetition rate $(80 \mathrm{MHz}$ and harmonics) was avoided. To measure the polarization-resolved RIN, a broad-band polarizer with a nominal extinction ratio of 1000: 1 was included. In addition, the VCSEL was tilted with respect to the collimation lens to avoid undesirable optical feedback that may increase the noise level.

\section{RESULTS AND DISCUSSION}

\section{A. Multimode Dynamics}

The VCSEL's present a multimode dynamics that is dependent on pumping conditions. At low pumping currents, the output of the devices is observed to be single mode. For a given prebias, as the amplitude of the pumping pulse $\left(I_{p}\right)$ is raised, higher order transverse modes start to oscillate with a turn-on delay that shortens with increasing $I_{p}$. This type of behavior is shown in Fig. 1 for an 18- $\mu \mathrm{m}$ VCSEL prebiased at $0.85 I_{\mathrm{th}}$. For $I_{p} \leq 0.6 I_{\mathrm{th}}$, the fundamental Gaussian mode $\mathrm{H}_{00}$ is dominant [Fig. 1(a)], but, when the amplitude of the pumping current is increased to $1.2 I_{\mathrm{th}}$, a higher order mode starts to lase after a delay of $T_{d} \approx 200 \mathrm{ps}$ [Fig. 1(b)]. This mode competes for the available gain and produces the extinction of the $\mathrm{H}_{00}$ mode. Transverse mode competition can result in mode hopping when the gain is not enough to sustain more than one mode, but it can also produce simultaneous multimode emission. The characteristic times for mode hopping were measured to be between 10 and 100 ps. At higher pumping currents, $I_{p}=4 I_{\text {th }}$, the turn-on delay of the second mode shortens and competition between higher order modes also occurs [Fig. 1(c)]. At even higher pumping currents, $9 I_{\mathrm{th}}$, almost all the modes are have been present since the beginning of laser action [Fig. 1(d)]. The turn-on delay of high-order transverse modes is also affected by the dc prebias. As expected, decreasing the prebias increases the turn-on delay of the modes.

\section{B. Dependence on the Active-Region Diameter}

VCSEL's with different active-region diameters showed different dynamics, since the boundary conditions of oscillating modes depend on cavity dimensions. Fig. 2 shows the spectrally and temporally resolved output of VCSEL's with different diameters prebiased at $0.85 I_{\mathrm{th}}$ and excited with 800-ps pulses with an amplitude $I_{p}=4 I_{\text {th }}$. The timeintegrated beam profiles of the different transverse modes in these conditions are plotted in Fig. 3. The corresponding contour maps are also shown to facilitate the identification of the different modes. Multimode operation is observed for all VCSEL's at this pumping condition, with the number of lasing modes increasing with increasing active-region size. Since the beam waist of the $\mathrm{H}_{00}$ mode increases with a square root dependence on the active-region diameter [19], larger VCSEL's have more gain available in the periphery, easing the threshold condition of higher order transverse modes. The wavelength separation between the modes was observed to be inversely proportional to the active region area, in agreement with previous theoretical studies [19], [20]. The total wavelength span of the spectra at the high pumping conditions capable of exciting all the modes is given by the gain spectrum and was measured to be of the order of $6 \AA$ for all diameters (Figs. 2 and 3).

For the 15- $\mu \mathrm{m}$ VCSEL [Fig. 2(a)], a higher order mode starts lasing after a delay $T_{d} \approx 200 \mathrm{ps}$ and then becomes dominant. This is the result of spatial hole burning of the carrier density in combination with a strong competition 


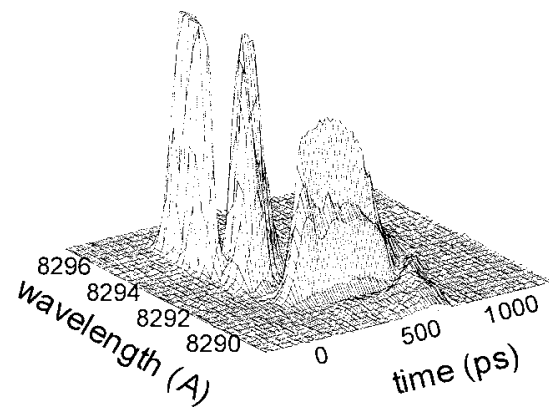

(a)

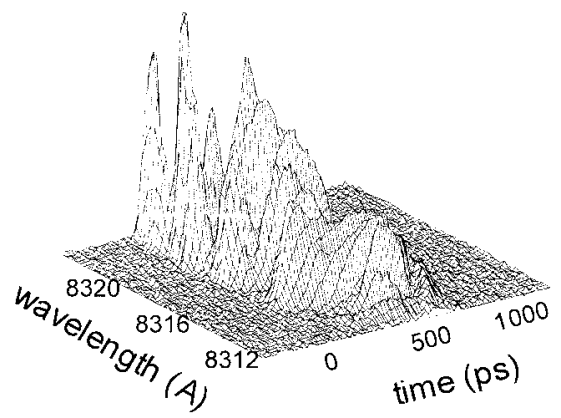

(b)

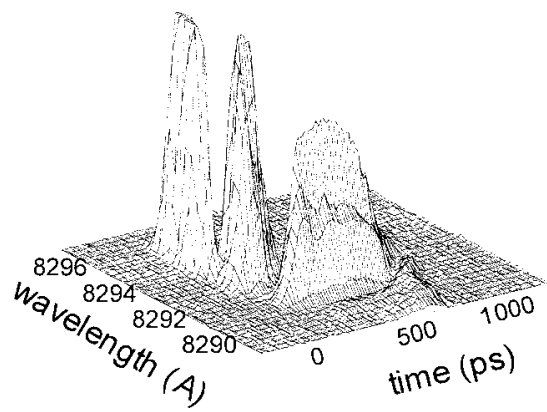

(c)

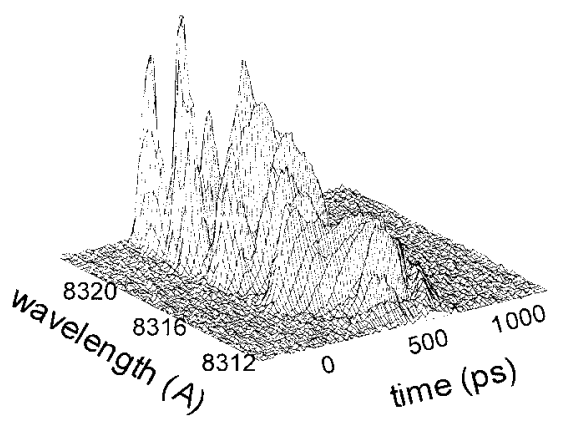

(d)

Fig. 1. Time-resolved spectra of a $18-\mu \mathrm{m}$ VCSEL prebiased at $0.85 I_{\text {th }}$ and excited with pulses of different amplitudes: (a) $0.6 I_{\mathrm{th}}$, (b) $1.2 I_{\mathrm{th}}$, (c) $4 I_{\mathrm{th}}$, and (d) $9 I_{\mathrm{th}}$. The observed change of the lasing wavelength is due to the heating produced by the average excitation power.

between the modes. As the field amplitudes of both modes have a large overlap, only one mode survives. The onset of a third mode is also observed after $T_{d} \approx 600 \mathrm{ps}$. A similar dynamics is observed for the 18- $\mu \mathrm{m}$ VCSEL [Fig. 2(b)], although up to three higher order modes were excited with onset delays of 40, 136, and $630 \mathrm{ps,} \mathrm{respectively.} \mathrm{For} \mathrm{the}$ 22- and 24- $\mu \mathrm{m}$ VCSEL's [Fig. 2(c) and (d)], the turn-on

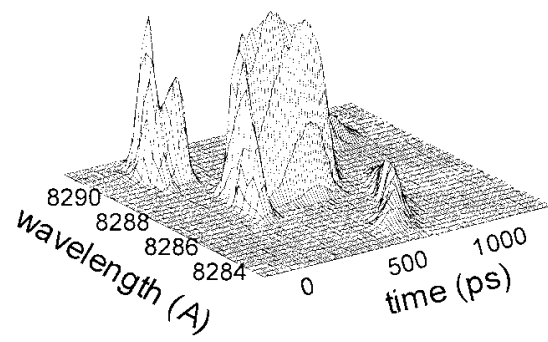

(a)

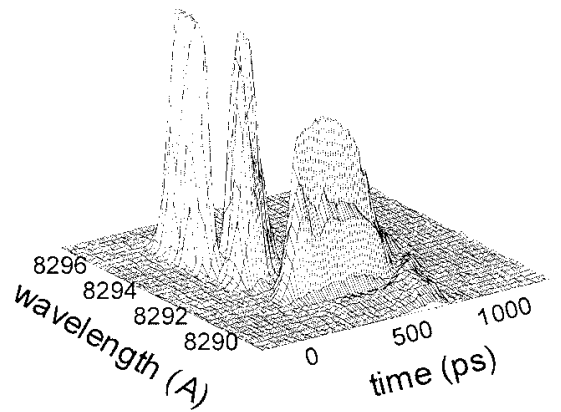

(b)

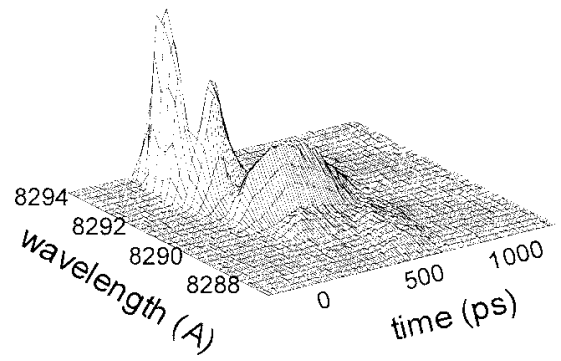

(c)

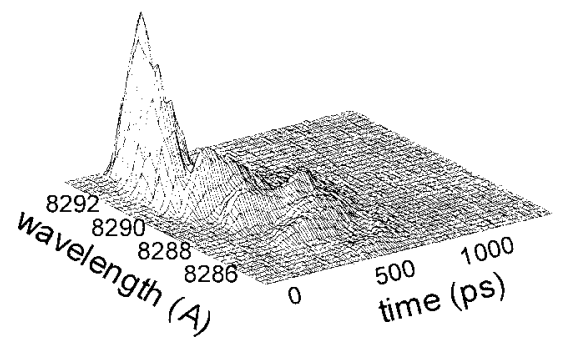

(d)

Fig. 2. Spectrally and temporally resolved output of VCSEL's with an active region diameter of (a) $15 \mu \mathrm{m}$, (b) $18 \mu \mathrm{m}$, (c) $22 \mu \mathrm{m}$, and (d) $24 \mu \mathrm{m}$. The devices were prebiased at $0.85 I_{\text {th }}$ and excited with pulses with an amplitude of $4 I_{\mathrm{th}}$.

delay of higher order modes is even shorter, giving rise to the simultaneous emission of several modes since the onset of laser action. It is usually the case that the first mode to lase is the Gaussian mode $\mathrm{H}_{00}$, although it was found that the 22- and 24- $\mu \mathrm{m}$ VCSEL's start lasing in a higher order transverse mode at pumping currents exceeding $4 I_{\mathrm{th}}$. Such behavior could be a direct consequence of the annular carrier injection profile which favors the excitation of higher order modes at high injection levels, while at current levels close to threshold diffraction losses prevent the onset of these modes.

The contour plots of Fig. 3 also reveal that the emission wavelengths present a rapid variation during the rise of the 


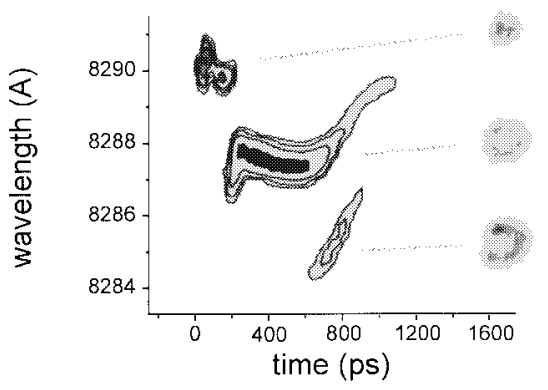

(a)

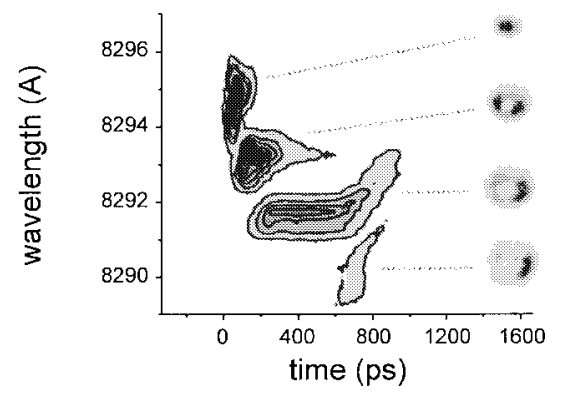

(b)

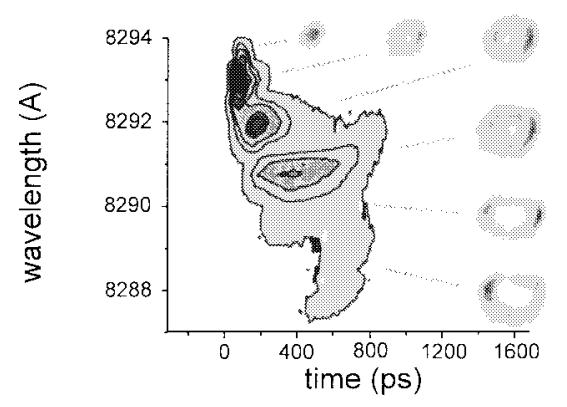

(c)

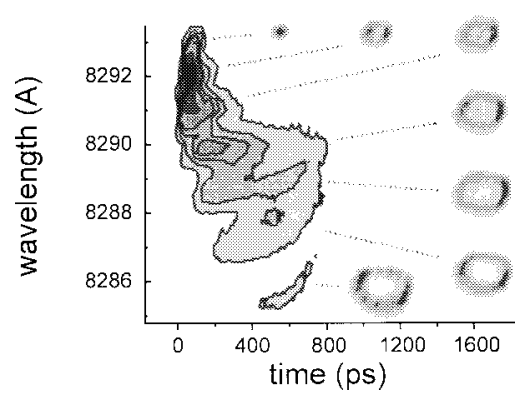

(d)

Fig. 3. Time-integrated modal beam profile for lasers with various active region diameters: (a) $15 \mu \mathrm{m}$, (b) $18 \mu \mathrm{m}$, (c) $22 \mu \mathrm{m}$, and (d) $24 \mu \mathrm{m}$. The contour maps of the transient response in the conditions of Fig. 2 are also shown.

stronger optical modes and a slower chirp that follows the trailing edge of the electrical pulse. Processes that account for these wavelength variations are self-phase modulation and the carrier density dependence of the VCSEL cavity length. Self-phase modulation accounts for the $\sim 2-\AA$ chirp at the beginning of laser emission [8]. However, the derivative of the photon number at the end of the pulse is too small to account for the slower chirp, which can be explained by means of the dependence of the refractive index on carrier density. The derivative of the refractive index with respect

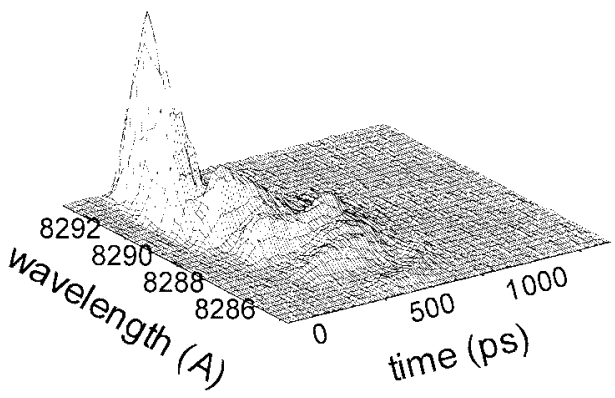

(a)

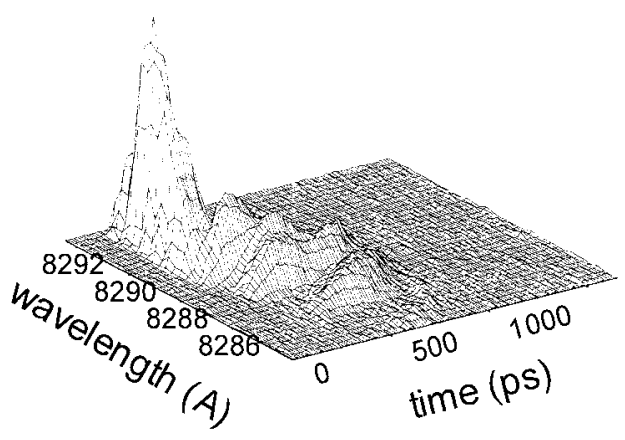

(b)

Fig. 4. Evolution of the output spectra of pulsed VCSEL's. (a) Parallel and (b) perpendicular polarization.

to the carrier density varies between -1.5 to $-5 \times 10^{-21}$ $\mathrm{cm}^{3}$, depending on whether the changes are due to free-carrier effects (above the bandgap) or bandgap renormalization (close to the bandgap) [21], [22]. Using these values, we obtain chirps of 1-3.5 $\AA$, in agreement with the experimental results.

\section{Polarization Characteristics}

In cylindrical symmetric VCSEL's, the laser emission has been found to be linearly polarized and aligned with the $\langle 011\rangle$ and $\langle 01 \overline{1}\rangle$ crystal axes [14]-[16]. In spite of the lack of polarization selection, one of these two states is usually dominant during $\mathrm{dc}$ operation. It has been shown that the characteristic time for establishing a dominant polarization state is of the order of 2-5 ns for a gain-guided VCSEL [16]. Polarization switching between these polarized states has also been reported during slow [15], [23] and fast [24] current ramp excitations. In our measurements, in which the excitation pulses had a duration of less than $1 \mathrm{~ns}$, both polarizations were present at the output of the VCSEL's. The multimode dynamics of these two polarization states is discussed next.

In order to record both polarizations, we included a half-wave plate after the VCSEL in the setup described in Section II. Because the sum frequency in the nonlinear crystal is polarization-sensitive, the polarization components of the laser output can be detected as the axis of the waveplate is varied with respect to that of the nonlinear crystal. We recorded the two orthogonal polarizations $P_{/ /}$and $P_{\perp}$, with $P_{/ /}$defined as the dominant polarization state during dc operation. Fig. 4 shows the evolution of the output spectrum of a 24- $\mu \mathrm{m}$ VCSEL with an excitation pulse of $4 I_{\mathrm{th}}$. It can be observed that not only are an equal number of modes excited 


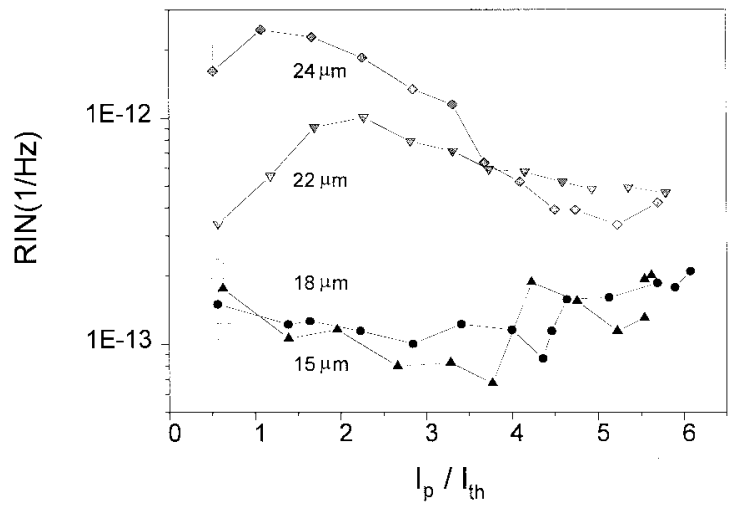

(a)

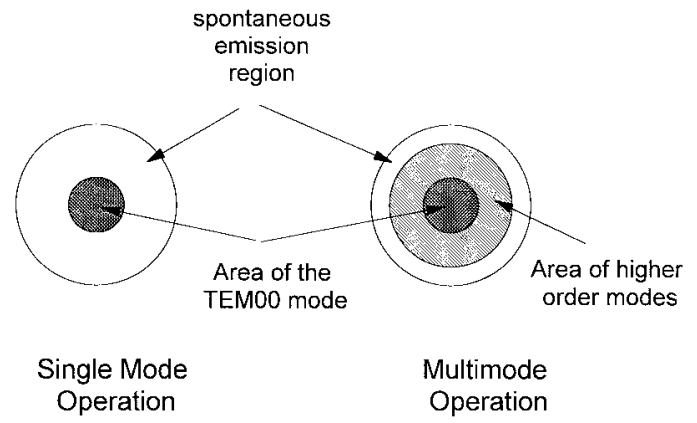

(b)

Fig. 5. (a) Dependence of the 200-MHz RIN on pumping current for VCSEL's of different sizes. (b) A diagram of the active region during single-mode and multimode operation is also included.

in both polarization states, but they evolve with the same dynamics. Within experimental error, the modes in the two polarization states have the same relative amplitude as well as the same turn-on delays. This behavior was observed in all the VCSEL's tested and indicates that, during fast excitation, both polarization states are isomorphic.

\section{Mode Dynamics and Noise}

The presence of higher order transverse modes oscillating in the laser cavity has been observed to limit the minimum attainable RIN during dc operation [25]. Consequently, the multimode dynamics discussed in the previous sections will have a strong influence on the noise levels of pulsed $\mathrm{VC}$ SEL's. In order to quantify this effect, we performed RIN measurements of the VCSEL output under the fast electrical excitation previously described.

The RIN spectra for VCSEL's of different active-region diameters showed a fairly flat frequency dependence for values up to $200 \mathrm{MHz}$. Above that frequency and up to $1.5 \mathrm{GHz}$, the RIN level decreases monotonically about one order of magnitude. Consequently, we concentrated on the low-frequency noise. The dependence of the 200-MHz RIN on pumping current is shown in Fig. 5(a). It can be observed that, at high pumping currents $\left(I_{p}>4 I_{\mathrm{th}}\right)$, the noise levels of the 22and 24- $\mu \mathrm{m}$ VCSEL's are similar and are only a factor of two higher than the RIN levels of the smaller devices. The behavior in the larger lasers can be understood in terms of a simplified picture of different modes acting as independent

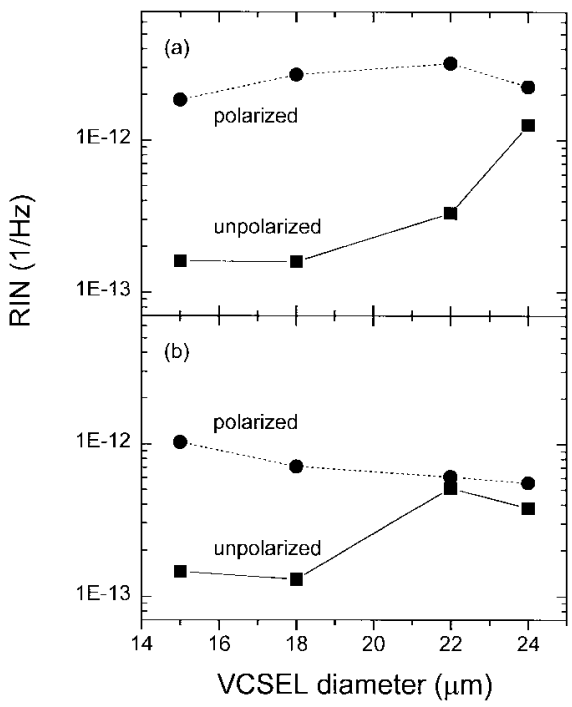

Fig. 6. Comparison between polarized and unpolarized RIN versus VCSEL size (a) for $I_{p}=0.6 I_{\mathrm{th}}$ and (b) for $I_{p}=6 I_{\mathrm{th}}$.

lasers, as illustrated in Fig. 5(b). At low pumping currents, the VCSEL's lase in single-mode or quasi-single-mode operation. In this case, the periphery of the active region, corresponding to the higher order modes, acts as a large source of spontaneous emission noise. On the other hand, at high pumping currents, simultaneous multimode operation is achieved and the volume of the spontaneous emission noise source is reduced. Our combined measurements of the multimode dynamics and noise levels clearly show that a noise reduction is achieved only during simultaneous lasing of the transverse modes.

Our measurements are also consistent with spontaneous emission noise being the main source of low-frequency RIN, in agreement with previous reports [16]. Hopping between the two lowest order modes was observed more clearly in the 15- and 18- $\mu \mathrm{m}$ VCSEL's, due to the higher threshold and the larger wavelength separation of the transverse modes. However, the presence of mode hopping could not be associated with a significant increase in the low-frequency RIN. From the spectrally and temporally resolved measurements shown in Fig. 1, the 18- $\mu$ m VCSEL's present mode hopping at $I_{p}=1.2 I_{\mathrm{th}}$, but the $200-\mathrm{MHz}$ RIN at this pumping condition remained around the same value found during single-mode operation $\left(\sim 10^{13} 1 / \mathrm{Hz}\right)$. Our time-resolved measurements presented in the previous section show that mode hopping is a fast process that takes place in $<100 \mathrm{ps}$ and is likely to affect the noise levels at high frequencies $(>10 \mathrm{GHz})$.

To evaluate the importance of the polarization fluctuations in multimode VCSEL's, we also measured the RIN spectra of the polarized output of the VCSEL's under study and compared them with the results presented previously. When polarized, the VCSEL's showed an increased lowfrequency RIN (Fig. 6). At low pumping currents $\left(I_{p}=\right.$ $\left.0.6 I_{\text {th }}\right)$, the polarization-resolved RIN is about $1-2 \times 10^{-12}$ $1 / \mathrm{Hz}$ for devices of different diameters [Fig. 6(a)], which represents an increase on the order of 10 times with respect to the unpolarized RIN of the smaller devices. For the larger VCSEL's, this increase was less important, suggesting that 
the spontaneous emission noise due to the large number of transverse modes in the cavity is far more important than energy partition between polarization states. On the other hand, at high pumping currents $\left(I_{p}=6 I_{\text {th }}\right)$, the polarizationresolved RIN showed a lower RIN for the larger VCSEL's [Fig. 6(b)]. At this pumping condition, several modes are lasing simultaneously in both polarization states, causing a reduction of the intensity noise as discussed earlier.

\section{ESTIMATION OF THE TURN-ON DELAYS IN LARGE-AREA VCSEL'S}

Inhomogeneities in the device structure and in the injection current cause asymmetries in the output intensity profile of VCSEL's, especially as the active region size is increased (see Fig. 3). In spite of this fact, VCSEL's can be considered to have a radially symmetric far-field intensity profile. This allows one to perform a detailed modeling of the radial distribution of carriers of small VCSEL's by using Laguerre-Gaussian modes to describe the optical field amplitude [8]. The turn-on dynamics and its influence in jitter and biterror rate can also be predicted utilizing statistical methods [26], [27]. However, a simpler approach can be utilized to estimate the delayed oscillation of high-order modes.

In contrast with edge emitters, the different modes in VCSEL's overlap only partially in the active region. To show the importance of this effect, we present next a simple model that treats each mode as an independent laser. Although crude, this approach reproduces the main features of the observed behavior. For a more accurate representation and quantitative predictions, important effects neglected in this model, such as the injected current profile and spatial hole burning, should be included.

The turn-on delay $T_{\mathrm{on}}$ of the output of a semiconductor laser as a function of the excitation pulse amplitude $(I)$ and the prebias current $\left(I_{o}\right)$ is given by [28]

$$
T_{\mathrm{on}}=\tau_{\mathrm{eff}} \ln \left(\frac{I-I_{O}}{I-I_{\mathrm{th}}}\right)
$$

where $\tau_{\text {eff }}$ is the effective differential carrier lifetime. Considering a similar dependence on current of the turn-on delay of the higher order modes, we find that the turn-on delay of mode $j$ with respect to the fundamental transverse mode $(j=0)$ is:

$$
T_{d}(j)=T_{\mathrm{on}}(j)-T_{\mathrm{on}}(0)=\tau_{\mathrm{eff}} \ln \left(\frac{\frac{I}{I_{\mathrm{th}}(0)}-1}{\frac{I}{I_{\mathrm{th}}(0)}-\frac{I_{\mathrm{th}}(j)}{I_{\mathrm{th}}(0)}}\right) .
$$

The threshold current, assuming a small transparency carrier density, can be approximated as

$$
I_{\mathrm{th}} \approx q\left(\Gamma g \tau \tau_{p}\right)^{-1}
$$

where $q$ is the electron charge, $\tau$ and $\tau_{p}$ are the carrier and photon lifetimes, $g$ is the gain coefficient, and $\Gamma$ is the transverse confinement factor calculated as the overlap integral of the gain area with the beam profile. Extending this

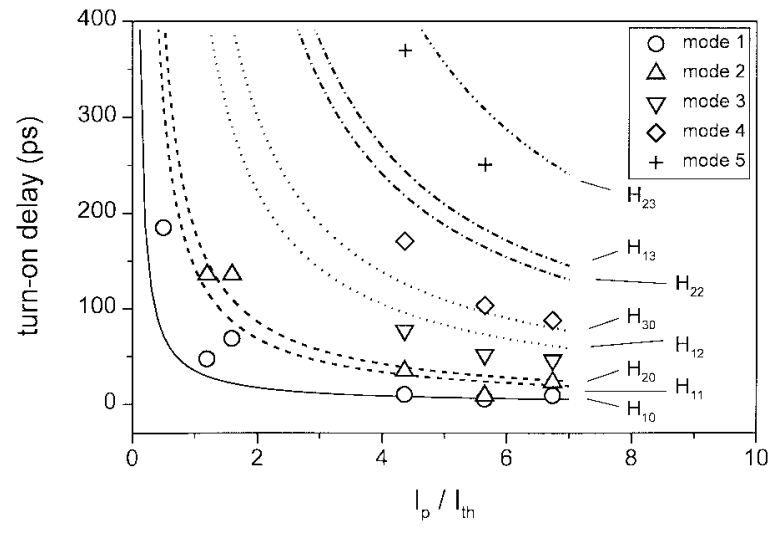

Fig. 7. Calculated turn-on delay of transverse modes in a $22-\mu \mathrm{m}$ VCSEL assuming Hermite-Gaussian modes $\mathrm{H}_{i j}$. The symbols represent the experimental points.

approximation to the different transverse modes, we can write the ratio of the threshold currents of modes $i$ and $j$ as

$$
I_{\mathrm{th}}(i) / I_{\mathrm{th}}(j) \approx \Gamma_{j} / \Gamma_{i}
$$

Using this result in (2) gives

$$
T_{d}(j)=\tau_{\mathrm{eff}} \ln \left(\frac{\frac{I}{I_{\mathrm{th}}(0)}-1}{\frac{I}{I_{\mathrm{th}}(0)}-\frac{\Gamma_{0}}{\Gamma_{j}}}\right) .
$$

The evaluation of the confinement factors requires the knowledge of the modal beam profiles. The mode profiles can be calculated from a full wave equation analysis such as that employed in [29], but, for the purpose of this calculation, it suffices to arbitrarily choose a set of transverse modes. We opted for Hermite-Gaussian modes $\left(H_{i j}\right)$, although similar results were obtained by using the Laguerre-Gaussian set. First, the waist size of the lower order mode $\left(\mathrm{H}_{00}\right)$ is determined from the VCSEL structure within the paraxial approximation, considering the hard mirror representation and "gain-aperturing" effect described in [19]. The injection current density was assumed to be uniform. This type of current distribution creates a parabolic temperature profile [30] that translates to thermally induced changes in the refractive index. This thermal lensing effect [31] was estimated from the prebias power. Due to the slow response of thermal effects $(\sim 2 \mu \mathrm{s})$ compared to the duration of the pulse and the small duty cycle of the pulsed excitation $(\sim 6 \%)$, only the dc portion of the input power was considered. From the resulting $\mathrm{H}_{00}$ mode diameter, the spatial distributions of the higher order modes and the confinement factors are determined.

Utilizing (5), the turn-on delays of the different transverse modes were calculated. Fig. 7 shows the calculated delays for the first eight higher order modes of the $22-\mu \mathrm{m}$ VCSEL as a function of pumping amplitude $I_{p}$. The calculations were performed considering an effective differential carrier lifetime of $1 \mathrm{~ns}$ and a dissipated power of $20 \mathrm{~mW}$ (the measured dc power at threshold). It should be noted that modes $H_{i j}$, in which the sum of the subindexes $i+j$ has the same value, also have the same wavelength and are plotted with 
the same line style in the figure. Fig. 7 also includes the experimental points for different transverse modes. As these modes were determined from their wavelength, they were numbered according to the value $i+j$ of the theoretical results. In this way, experimental mode 3 corresponds to the calculated modes $\mathrm{H}_{30}$ and $\mathrm{H}_{12}$.

Although this model utilizes mode profiles not completely accurate and assumes that the first mode to lase is always the $\mathrm{H}_{00}$ mode, it can be used to estimate the turn-on delay of the higher order modes in large-area VCSEL's. The agreement with experiment in VCSEL's with active-region diameters greater than $18 \mu \mathrm{m}$ is within $30 \%$. For the $15-\mu \mathrm{m}$ VCSEL's, the calculations underestimated the turn-on delays by a factor of $\sim 4$. This discrepancy is probably due to the fact that the overlap of the transverse modes is larger in smaller devices and our approximation of independent lasing modes is less accurate.

\section{SUMMARY}

We investigated the multimode response of VCSEL's with active-region diameters ranging from 15 to $24 \mu \mathrm{m}$ under subnanosecond electrical excitation. In all devices, the multimode dynamics exhibited a common feature of a delayed onset for the higher order modes that depends on the excitation conditions. This behavior is qualitatively different from that of edge emitters and is caused by the partial overlap of the different transverse modes in the active region. Due to this characteristic, a crude approximation of different transverse modes acting as independent lasers can be utilized to estimate the turn-on delay of higher order modes in large-area VCSEL's (diameter $\geq 18 \mu \mathrm{m}$ ). As expected, the number of modes excited increases with pumping power and, under similar conditions, is greater for larger VCSEL's. The richer multimode dynamics observed in the larger lasers correlate to a reduction of the noise characteristics of polarized and unpolarized devices. The simultaneous lasing of several transverse modes reduced the RIN in the larger VCSEL's and made them less sensitive to energy partition between polarization states. In addition, the two polarization states are present during subnanosecond excitation and evolve with the same dynamics.

\section{ACKNOWLEDGMENT}

The authors wish to acknowledge the contributions of G. Vashenko and thank Prof. S. A. Lee, Prof. C.-Y. She, and Prof. H. Temkin for lending part of the equipment used in this study.

\section{REFERENCES}

[1] Y.-M. Wong, D. J. Muehlner, C. C. Faudskar, D. B. Buchholz, M. Fishteyn, J. L. Brandner, W. J. Parzygnat, R. A. Morgan, T. Mullally, R. E. Leibenguth, G. D. Guth, M. W. Focht, K. G. Glogovsky, J. L. Zilko, J. V. Gates, P. J. Anthony, B. H. Tyrone, Jr., T. J. Ireland, D. H. Lewis, Jr., D. F. Smith, S. F. Nati, D. K. Lewis, D. L. Rogers, H A. Aispain, S. M. Gowda, S. G. Walker, Y. H. Kwark, R. J. S. Bates, D. M. Kutcha, and J. D. Crow, "Technology development of a highdensity 32-channel $16-\mathrm{Gb} / \mathrm{s}$ optical data link for optical interconnection applications for the optoelectronic technology consortium," J. Lightwave Technol., vol. 13, pp. 995-1013, 1995.

[2] R. Michalzik, P. Schnitzer, U. Fiedler, D. Wiedenmann, and K. J. Eveling, "High-bit-rate data transmission with short-wavelength oxidized
VCSEL's: Toward bias-free operation," IEEE J. Select. Topics Quantum Electron., vol. 3, pp. 396-403, 1997.

[3] C. J. Chang-Hasnain, M. Orestein, A. C. Von Lehmen, L. T. Florez, J. P. Harbison, and N. G. Stoffel, "Transverse mode characteristics of vertical-cavity surface-emitting lasers," Appl. Phys. Lett., vol. 57, pp. 218-229, 1990.

[4] C. J. Chang-Hasnain, J. P. Harbison, G. Hasnain, A. C. Von Lehmen, L. T. Florez, and N. G. Stoffel, "Dynamic, polarization and transverse mode characteristics of VCSEL's," IEEE J. Quantum. Electron., vol. 27, pp. 1402-1408, 1991.

[5] A. Valle, J. Sarma, and K. A. Shore, "Spatial hole burning effects on the dynamics of vertical cavity surface emitting lasers diodes," IEEE J. Quantum Electron., vol. 31, pp. 1423-1431, 1995.

[6] J. Martín-Regalado, S. Balle, and M. San Miguel, "Polarization and transverse-mode dynamics of gain-guided vertical-cavity surfaceemitting lasers," Opt. Lett., vol. 22, pp. 460-462, 1997.

[7] S. F. Yu, "Dynamic behavior of vertical cavity surface emitting lasers," IEEE J. Quantum Electron., vol. 32, pp. 1168-1179, 1996.

[8] O. Buccafusca, J. L. A. Chilla, J. J. Rocca, S. Feld, C. Wilmsen, V. Morozov, and R. Leibenguth, "Transverse mode dynamics in vertical cavity surface emitting lasers excited by fast electrical pulses," Appl. Phys. Lett., vol. 68, pp. 590-592, 1996.

[9] J. Y. Law and G. P. Agrawal, "Effects of spatial hole burning on gain switching in vertical cavity surface emitting lasers," IEEE J. Quantum Electron., vol. 33, pp. 462-468, 1997.

[10] H. Li, T. L. Lucas, J. G. McInerney, and R. A. Morgan, "Transverse modes and patterns of electrically pumped vertical-cavity surfaceemitting semiconductor lasers," Chaos, Solitons and Fractals, vol. 4, pp. 1619-1636, 1994.

[11] J. E. Epler, G. Gehsitz, K. H. Gulden, M. Moser, H. C. Sigg, and H. W. Lehmann, "Mode behavior and high resolution spectra of circularsymmetric GaAs-AlGaAs air-post vertical cavity surface emitting lasers," Appl. Phys. Lett., vol. 69, pp. 722-724, 1996.

[12] K. H. Hahn, M. R. Tan, Y. M. Houng, and S. Y. Wang, "Large area multitransverse mode VCSEL's for modal noise reduction in multimode fiber systems," Electron. Lett., vol. 29, pp. 1482-1483, 1993.

[13] K. H. Han, M. R. Tan, and S. Y. Wang, "Intensity noise of large area vertical cavity surface emitting lasers in multimode optical links," Electron. Lett., vol. 30, pp. 139-140, 1994.

[14] M. Shimizu, F. Koyama, and K. Iga, "Polarization characteristics of MOCVD grown GaAs/AlGaAs CBH surface emitting lasers," Jpn. J. Appl. Phys., vol. 27, pp. 1774-1775, 1988.

[15] K. D. Choquette, D. A. Richie, and R. E. Leibenguth, "Temperature dependence of gain-guided vertical-cavity surface emitting laser polarization," Appl. Phys. Lett., vol. 64, pp. 2062-2064, 1994.

[16] D. V. Kuksenkov, H. Temkin, and S. Swirhun, "Polarization instability and relative intensity noise in vertical cavity surface emitting lasers," Appl. Phys. Lett., vol. 67, pp. 2141-2143, 1995.

[17] J. Martín-Regalado, S. Balle, M. San Miguel, A. Valle, and L. Pesquera, "Polarization and transverse mode selection in quantum well vertical-cavity surface-emitting lasers: Index- and gain-guided devices," Quantum Semiclass. Opt., vol. 9, pp. 1-16, 1997.

[18] G. Cancellieri, Ed., Single-Mode Optical Fiber Measurement: Characterization and Sensing. Boston, MA: Artech House, 1993.

[19] D. I. Babic, R. J. Ram, J. E. Bowers, M. Tan, and L. Yang, "Scaling laws for gain guided vertical cavity lasers with distributed Bragg reflectors," Appl. Phys. Lett., vol. 64, pp. 1762-1764, 1994.

[20] R. Michalzik and K. J. Ebeling, "Generalized BV diagrams for higher order transverse modes in planar vertical cavity laser diodes," IEEE $J$. Quantum Electron., vol. 31, pp. 1371-1379, 1995.

[21] B. R. Bennet, R. A. Soref, and J. A. Del Alamo, "Carrier-induced change in refractive index of InP, GaAs, and InGaAsP," IEEE J. Quantum Electron., vol. 26, pp. 113-122, 1990.

[22] A. Olsson and C. L. Tang, "Injected-carrier induced refractive-index change in semiconductor lasers," Appl. Phys. Lett., vol. 39, pp. 24-26, 1981.

[23] K. D. Choquette, R. P. Schneider, Jr., K. L. Lear, and R.E. Leibenguth, "Gain-dependent polarization properties of vertical cavity lasers," IEEE J. Select. Topics Quantum Electron., vol. 1, pp. 661-666, 1995.

[24] J. Martín-Regalado, J. L. A. Chilla, J. J. Rocca, and P. Brusenbach, "Polarization switching in vertical cavity surface emitting lasers observed at constant active region temperature," Appl. Phys. Lett., vol. 70, pp. 3350-3352, 1997.

[25] D. M. Kuchta, J. Gamelin, J. D. Walker, J. Lin, K. Y. Lau, J. S. Smith, M. Hong, and J. P. Mannaerts, "Relative intensity noise of vertical cavity surface emitting lasers," Appl. Phys. Lett., vol. 62, pp. 1194-1196, 1993. 
[26] S. Balle, P. Colet, and M. San Miguel, "Statistics for the transient response of single mode semiconductor gain switching," Phys. Rev. A, vol. 43, pp. 498-506, 1991.

[27] J. Dellunde, M. C. Torrent, J. M. Sancho, and K. A. Shore, "Statistics of transverse mode turn-on dynamics in VCSEL's," IEEE J. Quantum Electron., vol. 33, pp. 1197-1204, 1997.

[28] R. W. Dixon and W. B. Joyce, "Generalized expressions for the turn-on delay in semiconductor lasers," J. Appl. Phys., vol. 50, pp. 4591-4595, 1979.

[29] M. Shimizu, F. Koyama, and K. Iga, "Transverse mode analysis for surface emitting laser using beam propagation method," IEICE Trans., vol. E74, pp. 3334-3340, 1991

[30] R. B. Bird, W. E. Stewart, and E. N. Lightfoot, Transport Phenomena. New York: Wiley, 1960.

[31] N. K. Dutta, L. W. Tu, G. Hasnain, G. Zydzik, Y. H. Wang, and A. Y. Cho, "Anomalous temporal response of gain guided surface emitting lasers," Electron. Lett., vol. 27, pp. 208-210, 1991.

O. Buccafusca (S'92-M'96), photograph and biography not available at the time of publication.
J. L. A. Chilla, photograph and biography not available at the time of publication.

J. J. Rocca, photograph and biography not available at the time of publication.

P. Brusenbach, photograph and biography not available at the time of publication.

J. Martín-Regalado, photograph and biography not available at the time of publication. 